\title{
PROGRAMA DE APRENDIZAJE-SERVICIO EN LA EDUCACIÓN SUPERIOR
}

\section{SERVICE-LEARNING PROGRAM IN HIGHER EDUCATION}

\author{
Eulalia Pino Loza, Ph.D.
}

Directora DIBESAU-Universidad Técnica de Ambato

eupino7@gmail.com

\author{
Washington Rojas \\ Mauricio Tamayo \\ Juan Castro
}

\section{RESUMEN}

El presente documento, analiza de manera cualitativa y cuantitativa las actividades de proyección social en la Universidad Técnica de Ambato (UTA), específicamente en las Facultades de Ciencias Humanas y de la Educación, Ciencias de la Salud, Jurisprudencia y Ciencias Sociales, para determinar y conocer cómo el conocimiento y la experiencia obtenida a través de acciones de servicio comunitario, coadyuva a la formación holística de los jóvenes estudiantes y a la solución de problemas del contexto. Para ello, mediante este estudio se indaga acerca de las expectativas de estudiantes, docentes y beneficiarios, las competencias desarrolladas, la oferta formativa, contenidos, metodología, recursos, formadores, proceso de enseñanza-aprendizaje, proceso de comunicación, objetivos, estructuras, experiencia obtenida, modelo de evaluación, dificultades encontradas, así como dirección y gestión en la educación universitaria, delimitando de esta forma, las habilidades cognitivas, afectivas, sociales y morales necesarias en el siglo XXI, para la formación de gestores de cambio que asuman como propósito del valor social del conocimiento, la participación en acciones solidarias y de servicio, vigorizando el fin humanista de la formación; la presente investigación analizó si la educación recibida empodera a los estudiantes para transformar la realidad, si existe impacto del modelo educativo de la UTA, expresada en la intervención social que mejora las condiciones de vida de la población beneficiaria. De esta manera, determinar si el aprendizaje-servicio es una herramienta que promueve la educación transformadora, fomenta habilidades para la vida con la existencia de una práctica consecuente, con la universidad esparcida en el compromiso, solidaridad y la responsabilidad social del estudiante.

Palabras clave: Aprendizaje-Servicio, educación transformadora, ciudadanía activa, competencias desarrolladas en la educación superior.

\section{ABSTRACT}

This paper analyzes qualitatively and quantitatively the social outreach activities at the Technical University of Ambato (UTA), specifically in the Faculty of Humanities and Education, Health Sciences, Law and Social Sciences, to determine and know how knowledge and experience gained through community service activities, contributes to the holistic education of young students and problem solving context. To do this, through this study inquires about the expectations of students, teachers and beneficiaries, developed skills, the training offer, content, methodology, resources, trainers, teaching-learning, communication process, objectives, structures, experience obtained evaluation model, difficulties encountered, as well as leadership and management in higher education, defining this way, cognitive, emotional, social and moral skills needed in the twenty-first century, for the formation of change managers assume purpose the social value of knowledge, participation in charity and service actions energizing the humanist end of training; this research examined whether the education received empower students to transform reality, if there is impact of the educational model of the UTA, expressed in social intervention that improves the living conditions of the beneficiary population. In this way, determine if the service-learning is a tool that promotes transformative education, promotes life skills with the existence of a consistent practice, the university spread on commitment, solidarity and social responsibility of the student.

Keywords: Service-learning, transformative education, active citizenship, higher education 


\section{Introducción}

El sistema educativo debe tener en cuenta la extraordinaria reserva de potencial espiritual y emocional que todo ser humano tiene y las posibilidades de emplearlo en la formación de futuros profesionales para el desarrollo sostenible de la humanidad. Una educación para la transformación implica, cambio personal en la manera de pensar, actuar y sentir, desarrollo de la capacidad intrínseca de sentir, de amar, así como, tener sensibilidad ante las necesidades y sentimientos de los demás. Cambio social facilitando una praxis social transformadora de estructuras que promueven una sociedad justa, pacífica y equitativa. Cambio Ambiental, en relación con el cuidado y conservación de los recursos naturales.

El ser humano crea el mundo exterior a imagen y semejanza de su mundo interior, las actitudes y comportamientos se generan a partir de la concepción que tiene el ser humano de sí mismo y del mundo. Tenemos el mundo que tenemos porque tenemos la educación que tenemos, la educación es nuestra mejor esperanza para evolucionar personal y socialmente. Solo una educación con enfoque humanista puede transformar la sociedad, necesitamos una educación para trascender, dejar atrás modos de pensar y vivir obsoletos, educar para la vida en sociedad con mejor salud emocional y existencial (1).

Aprendizaje-servicio es una herramienta educativa que permite el desarrollo holístico del estudiante como persona completa. Se fundamenta en un conocimiento conceptual integrado, basado en los procesos de pensamiento, en el desarrollo emocional, social y moral, fomenta habilidades para la vida como la comprensión, el respeto, y fortalece el desarrollo de una conciencia profunda y empática. (2)

Este marco conceptual es el fundamento de la presente investigación relacionada con el aprendizaje-servicio que analiza la percepción de los/ las estudiantes respecto a esta temática, cómo se han fortalecido sus habilidades para el desempeño, sus bases teórico-prácticas, el uso de herramientas psicopedagógicas, didácticas, asesoramiento recibido, principales problemas detectados y el impacto final de esta práctica educativa.

\section{Dimensión Social de la Educación en el siglo XXI}

Nos encontramos ante una época de profundas crisis. Crisis en lo económico, en el ámbito político y en lo social. La sociedad está en crisis, esa situación se refleja evidenciando los problemas en el mundo como son la destrucción del medioam- biente, incremento de desigualdades sociales y degradación de la psique humana(1). No debemos obviar que unido a cada momento de crisis o depresión socio-económico cabe la posibilidad de ver emerger nuevos horizontes que permitan y nos lleven a un cambio de modelo social, asumiendo la premisa de que cada revolución socio-económica, si analizamos la historia del mundo occidental, lleva implícita un nuevo salto en la cognición humana. Las necesidades o demandas para este Siglo XXI centran su atención en el impulso de la ciencia para la transformación tecnológica, búsqueda de nuevos perfiles profesionales, institucionalización, desarrollo y expansión de la actividad científica- socioeconómica y cultura (3).

En lo que se refiere a la educación superior universitaria, todas las instituciones fomentan la diversificación de estudios superiores de carácter técnico, en la búsqueda de una universidad que sea partícipe e influya en el desarrollo socio-económico a través de su vinculación con el sistema productivo. Algunos autores sitúan la actual problemática en la revisión de la eficiencia académica del currículo universitario(4).

En este siglo XXI la educación se ha convertido en la principal ventaja competitiva de las naciones. El capital humano está desplazando al capital físico y al capital financiero como verdadero generador de riqueza en el futuro (5). A nuestro entender, las funciones sociales de la universidad son: la formación holística de su comunidad, la investigación, la docencia, la proyección social, gestión, emprendimiento e innovación. A la formación holística le corresponde principalmente en el siglo XXI formar personas íntegras, acorde al perfil profesional holístico, con competencias afectivas, morales y sociales, dimensiones personal, social, profesional y laboral (6). (7)Orozco 2010, otra función social dependiente de la investigación y la docencia, es la formación de la actitud crítica. Por medio de ella se cuestiona la cultura, los fenómenos y los valores de su tiempo, con lo cual se crea, se promueve y se eleva la cultura misma. Dentro de la educación holística y con enfoque de la persona completa (8) Covey 2005, considera cuatro dimensiones imbricadas: inteligencia mental, emocional, existencial y física.

La esencia de la educación, es la transmisión de principios y valores, pero estos no nos ayudan a escoger nuestro sendero en la vida hasta que los hagamos propios, y sean una parte de nuestro modelo mental. Esto significa que son más que simples fórmulas o aseveraciones dogmáticas; significa que pensamos y sentimos con ellos, que llegan a ser los mismos instrumentos, por los cuales miramos, interpretamos y experimentamos el 
mundo. Cuando pensamos, no simplemente pensamos; pensamos con ideas (9).

La Universidad desde sus comienzos se configura como una institución de Educación con una triple función en la sociedad: función cultural, investigadora y social. Históricamente la educación ha sido un proceso de actuación intencional sobre miembros de una comunidad humana, principalmente sobre los más jóvenes, con el propósito de desarrollar su personalidad, capacitarlos para el trabajo y adaptarlos a la vida social. Es un proceso que acompaña a las sociedades durante toda su evolución y que, de alguna manera, refleja las cambiantes estructuras de dichas organizaciones, sus contradicciones y problemas, su crecimiento y diversificación constantes, el proceso de evolución social, las fuerzas productivas, la ciencia, la tecnología y en general la cultura(10).

La educación es nuestra mejor esperanza para cambiar el mundo, una educación para la transformación que nos ayude a evolucionar personal y socialmente. Es evidente que existe crisis en los procesos de generación y aplicación del conocimiento, porque vivimos en un sistema deshumanizado, automatizado, globalizado que controla el dinero, modas, propagandas intereses de grupos, discusión fanática, poder político y económico. La necesidad del siglo XXI requiere una visión unificadora del hombre, sociedad, naturaleza. La universidad es una institución generadora de realidades socioculturales y pensamiento propio transformador y con proyección hacia la vida social.

La enorme energía que nos proporciona el conocimiento será consagrada a aquellos fines que extiendan el alcance de las investigaciones humanas y el desarrollo tecnológico, al aumento de la productividad de la humanidad, a la extensión de la investigación científica, a la prolongación de la vida humana y al fomento de cualquier otro agente que pueda estimular la vida intelectual, moral y espiritual de toda la raza humana.

El ingreso del siglo XXI marca la necesidad de un profesional capaz de relacionarse con la complejidad, nuestros profesionales, además de poseer rigor y profundidad en el dominio de un campo del conocimiento, deberán tener un entendimiento del universo, de su país, de su región, de su cultura, de sí mismos, deberán desarrollar una visión crítica y creativa frente a los procesos de generación y circulación del conocimiento, que les permita valorar tanto los modelos matemáticos y experimentales propios de las ciencias básicas, como los modelos de la comprensión y de la acción social representativos de las ciencias sociales y humanas, deberán comprender y pensar sistémicamente sobre los problemas morales y éticos, poseer criterios de orden estético, rechazar en todas sus formas la falsedad, deberán tener la capacidad de pensar y escribir con claridad, argumentar racionalmente y hacer uso del lenguaje en el foro público (6)

En las últimas décadas las universidades en general se han preocupado principalmente por construir el conocimiento científico y por desarrollar las habilidades y destrezas relacionadas con el campo profesional desde un enfoque disciplinar; pero se ha descuidado la parte más formativa del hombre: la construcción de sus valores éticos, morales y sociales; pues el hombre, con el pasar de los años, pone de manifiesto su calidad de "persona" y los valores que gobiernan su vida. Por lo tanto para que dichos valores se manifiesten, sobre todo en el ejercicio de sus profesiones, es necesario que estos sean cimentados en todos los niveles de la educación, sobre todo en el Superior(11).

\section{El aprendizaje-servicio, un instrumento de la educación para la ciudadanía}

La ciudadanía es la fuente del vínculo social, y es ejerciendo los derechos y las prácticas de la ciudadanía como los individuos forman una sociedad. Las enseñanzas aportan a la transformación:

La transformación individual de cada persona, con el fin de crear una nueva calidad de hombres comprometida con la práctica de cualidades morales; y,

La transformación de la sociedad, con el fin de aportar a una nueva civilización caracterizada por la unidad en diversidad, la justicia, un compromiso con el bienestar común y la paz universal.

Según (12)Serra Vásquez 2010: aprendizaje-servicio: ... "es un enfoque pedagógico" basado en una experiencia de servicio solidario, en la cual los estudiantes, docentes y miembros de la comunidad aprenden, se forman y trabajan juntos para satisfacer necesidades comunitarias, potenciándose mutuamente los objetivos curriculares de un curso con los objetivos del servicio. El que sea un enfoque pedagógico, hace referencia a que Aprendizaje Servicio es más que una metodología en la que los docentes, estudiantes y socios comunitarios deben dominar un conjunto de conocimientos, procedimientos y habilidades, para alcanzar con éxito los objetivos.

La gran revelación del aprendizaje-servicio, en 
forma de experiencia vivida, es que aprender, sirve; y servir, enseña. El aprendizaje-servicio no representa una novedad absoluta, sino una combinación original de dos elementos conocidos: el aprendizaje basado en la experiencia y el servicio a la comunidad. Aprendizaje-servicio considera nuevos roles y relaciones para los tres actores directamente involucrados: el estudiante como protagonista, el docente como transformador social, y la contraparte como socio comunitario.

El Programa de aprendizaje-servicio en la formación superior permitiría: a) desarrollar en los estudiantes los valores de la solidaridad y el compromiso con el bienestar colectivo; b) integrar el conocimiento teórico práctico aprendido en las aulas con una experiencia cognitiva, desarrollada de manera interdisciplinaria y en contacto con las problemáticas más acuciantes del país; c) profundizar la relación entre la Universidad y los problemas nacionales, permitiendo generar un vínculo de retroalimentación entre las funciones de proyección social, docencia e investigación, que facilitará readecuar los contenidos curriculares y las tareas de investigación en función de las necesidades y desafíos del proceso de desarrollo nacional; d) la democratización crecientemente de la Universidad: democratización de saberes y tecnologías, democratización de las formas de construcción de esos saberes a partir de incorporar los saberes y experiencias populares, democratización a partir de reestructurar la Universidad en base a las necesidades nacionales, democratización a partir de integrar al conjunto de la comunidad universitaria y a la comunidad toda, al proceso de elaboración de una universidad nueva; e) promover el trabajo interdisciplinario, como forma de abordaje de la realidad; f) desarrollar conocimiento y tecnologías vinculadas al desarrollo nacional, ejerciendo una verdadera soberanía cognitiva o autonomía científica; g) promover la planificación estratégica del Sistema de Educación Superior, detectando las demandas nacionales y orientándose en función de las mismas, a la vez que incorporando a los distintos actores sociales y estatales en el diagnóstico, elaboración y ejecución de las distintas políticas (12).

Este tipo de proyectos tienen doble intencionalidad. En primer lugar, está la intencionalidad pedagógica (aprendizaje), a través de la cual se busca posibilitar mejores aprendizajes, tanto en la dimensión cognitiva, procedimental y actitudinal. No sólo del estudiante, sino también los docentes (quienes son con-responsables de catalizar procesos de aprendizaje en los demás actores) y los socios comunitarios. Intencionalidad solidaria que implica asumir valores ligados a la justicia y promoción de los derechos humanos, fortalecer actitudes pro-sociales, la resiliencia que los estudiantes asuman compromisos en función del bien común(13).

Tres procesos relacionales son el producto de la afirmación anterior. En primer lugar, el proceso de transferencia de conocimientos ligado a la dimensión cognitiva, a través del cual los distintos actores comparten sus saberes, ya sean académicos, de experticia o de vida. En este sentido, cada uno de los actores es "educador-educando", teniendo un saber (y un no saber) precioso para los otros; y a la vez es un "educando-educador", pudiendo aprender del otro.

Un segundo proceso es el de empoderamiento, ligado a la dimensión procedimental, a través del cual los distintos actores participan, de manera activa y crítica, de las decisiones y acciones que comprenda el proyecto, a través de esta participación, los distintos actores irán comprendiendo y aprehendiendo las herramientas y recursos necesarios para poner en práctica los aprendizajes. El tercer proceso es el de Integración Social, ligado a la dimensión valórico-actitudinal, a través del cual los distintos actores se van formando en valores solidarios. Esto significa que cada uno de los actores toma conciencia de las necesidades del otro, y desarrolla un aprendizaje en torno a cómo trabajar tanto la necesidad específica de la comunidad así como la necesidad del otro.

\section{Nueve Competencias o Dominios}

Según (2) Huitt W. y Ratzke de Figueiredo 2011: son 9 competencias o dominios que se articulan en los planes de estudio y análisis, que determinan la estructura para el desarrollo holístico: El conocimiento conceptual, académico integral, los procesos de pensamiento, desarrollo emocional y social, carácter moral y su aplicación en proyectos de Aprendizaje-Servicio:

1. Auto concepto: construcción de sí mismo, persona equilibrada, reflexiva, comprometida, con fluidez.

2. Cognición -Pensamiento-Inteligencia: persona bien informada, intelectual.

3. Emoción- Inteligencia afectiva: persona emocionalmente desarrollada, tiene conciencia de sus propias emociones y conexión empática con los demás desarrolla el optimismo, desarrolla la gratitud, cuidado.

4. Volitivo-Voluntad: capacidad para originar una acción intencionada, controla sus acciones, hace correcciones para alcanzar sus propias metas, 
son personas indagadores, tomadores de riesgo, resilientes.

5. Física-Kinestésica: consciente de su cuerpo en el espacio y el movimiento, poseen estilo de vida saludable, capacidad física básica.

6. Espiritualidad-Propósito: capacidad de generar significado y propósito para la vida, de crear una relación profunda y personal con uno mismo, con los demás, con la naturaleza y las incógnitas universales, impulso creativo, instar a la trascendencia.

7. Social-Interpersonal: habilidad para tratar con otras personas, servicio social, empatía, personas comunicadoras, con habilidad interpersonal, prosociales.

8. Carácter moral: capacidad para desarrollar hábitos y patrones de pensamiento, las emociones, intenciones y comportamientos asociados con el bien y el mal, especialmente en un contexto social, desarrollo de sensibilidad ética, juicio ético, motivación ética, acción ética, aplica principios, valores y virtudes.

9. Ciudadanía: desarrollo de la conciencia sociocultural (mente abierta), valoran estructuras sociales, roles de adultos, participación activa.

\section{Necesidades y Contexto de Intervención}

En este estudio se razona sobre la necesidad de crear el programa de aprendizaje-servicio, cuyo eje central es la formación en valores, resiliencia, ciudadanía activa, prosocialidad como habilidades primordiales para el desarrollo del carácter moral del individuo, que debe gestionarse con los elementos teóricos y prácticos. Con la certeza de que la educación holística se consigue no solo con el desarrollo del conocimiento a través del estudio de las ciencias, sino también a través de la realización de acciones solidarias y de servicio.

En el Ecuador se vive un proceso de transformación desde el año 2007, una vez que el gobierno de la revolución ciudadana implementa diversos instrumentos, como la Constitución aprobada en el año 2008, el Plan Nacional de Desarrollo 200920013. En materia educativa establece un nuevo régimen de desarrollo basado en un sistema de igualdad de oportunidades y justicia social, que tiene como objeto y fin el desarrollo del ser humano. Por otro lado, el régimen del Buen Vivir establece el sistema nacional de inclusión y equidad social, que es el conjunto articulado y coordinado de sistemas, instituciones, políticas, normas, programas y servicios que aseguran el goce de los derechos.

El proyecto de cambio para el Buen Vivir se encamina a construir una sociedad que reconozca la unidad en la diversidad, reconocer al ser humano como gregario que desea vivir en sociedad, promover la igualdad, la integración y la cohesión social como pauta de convivencia, garantizar progresivamente los derechos universales y la potenciación de las capacidades humanas, construir relaciones sociales y económicas en armonía con la naturaleza, edificar una convivencia solidaria, fraterna y cooperativa, consolidar relaciones de trabajo y de ocio liberadores, reconstruir lo público, profundizar la construcción de una democracia representativa, participaba y deliberativa, y consolidar un estado democrática, pluralista y laico (14).

La finalidad de esta investigación es analizar el desarrollo y aplicación de principios y valores en las actividades de proyección social de la Universidad Técnica de Ambato con la intención de proponer la implementación de un modelo de Universidad basado en la metodología de aprendizaje-servicio. Se pretende motivar a la comunidad universitaria y lectores sobre la necesidad imperiosa de dar atención a un problema mundial como es la "crisis de valores", porque es tarea ineludible de las instituciones educativas vigorizar la acumulación de conocimiento a través del estudio intelectual en las diversas disciplinas sino también el desarrollo y aplicación de principios y valores.

Población

\begin{tabular}{|c|c|c|c|c|c|c|}
\hline \multirow{3}{*}{ Facultad } & \multicolumn{4}{|c|}{ POBLACIÓN UNIVERSO } & \multirow{2}{*}{\multicolumn{2}{|c|}{ Rango edad }} \\
\hline & & $\%$ estudiantes de la & & & & \\
\hline & N Estudiantes & & $\%$ Mujeres & $\%$ Hombres & 8 a 25 años & >25 años \\
\hline $\begin{array}{l}\text { Jurisprudencia y } \\
\text { Ciencias Sociales }\end{array}$ & 762 & $5,2 \%$ & $50 \%$ & $50 \%$ & \multirow{3}{*}{$75 \%$} & \multirow{3}{*}{$25 \%$} \\
\hline $\begin{array}{l}\text { Ciencias Humanas y } \\
\text { de la Educación }\end{array}$ & 4.478 & $30,6 \%$ & $70 \%$ & $30 \%$ & & \\
\hline Ciencias de la Salud & 2.063 & $14,1 \%$ & $70 \%$ & $30 \%$ & & \\
\hline
\end{tabular}

Tabla a: elaborada por autores 
Se seleccionaron, acorde a población estudiantil, a 10 docentes de la Facultad de Jurisprudencia y Ciencias Sociales 15 de la Facultad de Ciencias de la Salud y 15 docentes de la Facultad de Ciencias Humanas y de la Educación en total 40 docentes considerando a tutores de actividades de vinculación con la colectividad, coordinadores de las carreras y autoridades de las Facultades. En cuanto a los beneficiarios, se determinaron acorde a los Proyectos realizados por cada una de las Facultades.

\section{Muestra}

De la Facultad de Ciencias Humanas y de la Educación, 194 estudiantes de ocho carreras, de la Facultad de Ciencias de la Salud, 192 estudiantes de seis carreras, de la Facultad de Jurisprudencia y Ciencias Sociales, 90 estudiantes de tres carreras, modalidad presencial. La muestra de docentes se conformó por 40 profesores involucrados en proyectos de vinculación social, que han participado como asesores de los estudiantes, y autoridades de la Universidad Técnica de Ambato. Para el estudio con los beneficiarios, se identificó una muestra de 400 usuarios de los programas de vinculación que se vienen desarrollando en comunidades pertenecientes a los cantones de la provincia de Tungurahua. Los datos fueron tomados a beneficiarios de 6 proyectos realizados por cada Facultad. Excepto la Facultad de Ciencias de la Salud que implementaron 4 proyectos.

\section{Objetivos e hipótesis}

El objetivo general de esta investigación es la necesidad de crear un Programa de aprendizaje-servicio en la Universidad Técnica de Ambato, como modelo pedagógico para la ejecución de actividades de proyección social.

La hipótesis de partida es que el cumplimiento de las expectativas en la experiencia comunitaria fortalece las competencias personales, académicas y laborales del estudiante lo que le permite desempeñarse y enfrentarse satisfactoriamente al mundo del trabajo.

Como objetivos específicos se busca, investigar de una manera minuciosa cuales son los principales factores que intervienen en las actividades de proyección social. Descubrir si estos tienen una relación directa en la formación, calidad y/o bondad, fin esperados en la comunidad. Evaluar como los contenidos académicos y axiológicos de la educación recibida o adquirida por los y las jóvenes estudiantes influyen en los resultados obtenidos y diagnosticar la situación actual del programa de Vinculación con la Colectividad de la
Universidad Técnica de Ambato.

\section{Metodología}

La investigación se desarrolló desde un planteamiento metodológico mixto, cualitativo-cuantitativo por ser el apropiado para el objeto de estudio, se enmarcó dentro de las fases: exploratoria y descriptiva, que permitieron ir analizando y relacionando variables que impactan positiva y negativamente sobre la estructura que dispone la UTA para la realización de las actividades de proyección social o vinculación con la sociedad. Dentro del enfoque cuantitativo los planteamientos a investigar fueron específicos y delimitados desde el inicio del estudio. Desde este enfoque la observación y encuestas permitieron delimitar las características del problema y posibles soluciones, establecer resultados relevantes y verificar hipótesis. En el enfoque cualitativo, la observación y entrevista permitieron conocer las causas del problema y las posibles soluciones para mejorar las actividades de proyección social.

\section{Técnicas de investigación}

En cuanto a las técnicas de investigación se utilizó la técnica documental que permitió la recopilación de información, para enunciar las teorías que sustentan este estudio. El marco referencia se basa principalmente en experiencias de otros países latinoamericanos con amplio conocimiento en programas y proyectos de vinculación con la sociedad. Y la técnica de campo que permitió el contacto directo con la población objetivo, y el acopio de testimonios que permitieron confrontar la teoría con la práctica, en la búsqueda de la verdad objetiva.

\section{Procedimiento}

Previo a la aplicación de la encuesta se realizó una selección y validación de las preguntas del cuestionario. El estudio se realizó en el período marzo a agosto de 2010, con entrevistas a docentes de las tres Facultades. Posteriormente, se realizó la investigación cuantitativa, mediante la aplicación de un cuestionario estructurado a los actores de esta investigación: docentes, estudiantes y beneficiarios, para luego procesar y analizar la información. Se utilizó el programa estadístico SPSS (Statistical Package for the Social Sciences) en su versión 18, para el correspondiente análisis.

\section{Resultados y discusión}

Actividades de proyección social y la formación para el trabajo profesional 
En la Tabla1, el $66.8 \%$ de los estudiantes indican que las actividades de proyección social en la UTA, se enfocan a la formación del perfil profesional en lo intelectual, en menor proporción en lo social y personal, sin profundizar en su formación para el desenvolvimiento laboral sin dar importancia a las experiencias comunitarias, el $65 \%$ de profesores manifiestan que existe relación con la formación para el trabajo profesional, que las actividades de vinculación son un soporte para la relación científica, tecnológica, cultural como función integral de la educación, profesionalización y formación para el trabajo.

Tabla 1.Relación entre actividades de proyección social y formación laboral

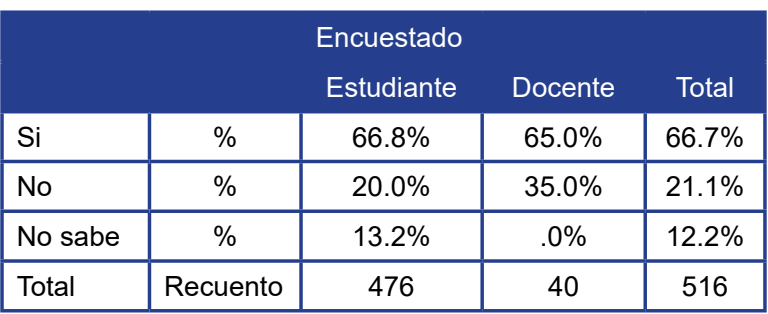

Elaborado por autores

Fuente: Docentes, estudiantes de la Facultades de Ciencias Humanas,

Ciencias de la Salud, Jurisprudencia y Ciencias Sociales.

Formando mujeres y hombres, calificados, con dimensión holística comprometidos con valores que defienden y difunden activamente; que ven su profesión como una posibilidad de servicio a los demás, que son capaces de aportar como ciudadanos a la construcción de la sociedad y de responder creativamente a los desafíos de un proyecto país(15).

Por otro lado, el análisis de la hipótesis a un nivel de significancia $\alpha 0,05$, el p-valor es 0,280 por lo cual se acepta la hipótesis. Resultado que concuerda con lo mencionado por (6)Morales (2011): Fortalecer la formación holística, sistémica por procesos, es considerar a las ciencias sociales como instrumentos útiles para la liberación humana, los conocimientos científicos en el campo de las ciencias humanas tengan una aplicación práctica y sirvan para encontrar soluciones a los problemas sociales, para realizar acciones concretas orientadas a mejorar la calidad de vida(Figura 1).

\section{Figura 1. Relación del cumplimiento de} expectativas y el desempeño laboral

El cumplimiento de expectativas y formación recibida por el estudiante permite desempeñarse satisfactoriamente en el mundo del trabajo

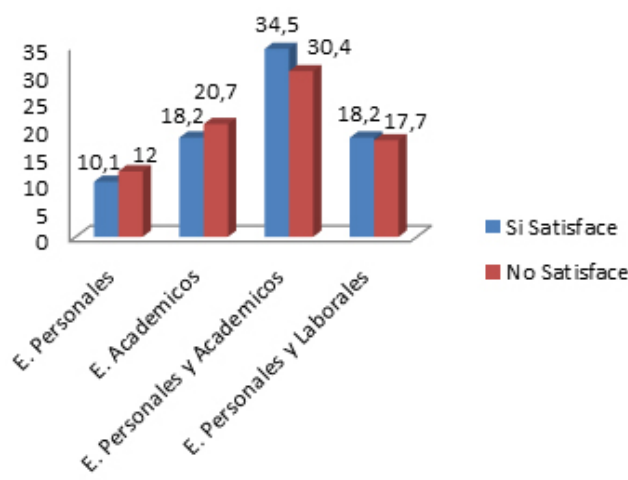

Elaborado por autores

Fuente: Docentes, estudiantes de la Facultades de Ciencias Humanas, Ciencias de la Salud, Jurisprudencia y Ciencias

Sociales

Relación de las actividades de proyección social y el Plan Nacional con el ejercicio de la futura profesión

No existe un fin trascendente un producto importante, como una propuesta de intervención o proyecto sostenible, sistematización de experiencias; es decir, resultados de un equipo de profesionales, trabajo multidisciplinario, de impacto tanto para la universidad como para las comunidades o diferentes lugares de actuación(16).

En relación a los resultados, la mayoría de alumnos $81,7 \%$ y docentes $65 \%$, manifiestan que como resultado final presentan un informe que no es analítico ni propositivo, en cuanto a los usuarios un $58,8 \%$ responde que no sabe, es decir no se construye con los beneficiarios la solución de sus propios problemas, solo se realizan desde la Universidad (Tabla 2).

Tabla 2. Resultado final de las actividades de proyección social

\begin{tabular}{|c|c|c|c|c|c|}
\hline \multirow[b]{2}{*}{ Facultad } & \multicolumn{4}{|c|}{ Encuestado } & \multirow[b]{2}{*}{ Total } \\
\hline & $\mathrm{N}^{\circ}$ Estudiantes & Est. & Docente & Usuario & \\
\hline Propuesta & $\%$ & $10,9 \%$ & $10,0 \%$ & $12,3 \%$ & $11,5 \%$ \\
\hline Informe & $\%$ & $81,7 \%$ & $65,0 \%$ & $29,0 \%$ & $58,0 \%$ \\
\hline Sistematización de experiencias & $\%$ & $7,4 \%$ & $15,0 \%$ &, $0 \%$ & $4,5 \%$ \\
\hline Publicación de resultados & $\%$ &, $0 \%$ & $5,0 \%$ &, $0 \%$ &, $2 \%$ \\
\hline $\begin{array}{l}\text { Implementación de un proyecto } \\
\text { sostenible }\end{array}$ & $\%$ &, $0 \%$ & $5,0 \%$ &, $0 \%$ &, $2 \%$ \\
\hline No contesta & $\%$ &, $0 \%$ &, $0 \%$ & $58,8 \%$ & $25,7 \%$ \\
\hline Total & Recuento & 476 & 40 & 400 & 916 \\
\hline
\end{tabular}

Elaborado por autores Fuente: Docentes, estudiantes de la Facultades de Ciencias Humanas, Ciencias de la Salud, Jurisprudencia y Ciencias Sociales 
Incorporar el enfoque de Aprendizaje-Servicios que se basa en una experiencia de servicio solidario, en la cual estudiantes, docentes y miembros de la comunidad aprenden, se forman y trabajan juntos para satisfacer necesidades comunitarias, potenciándose mutuamente los objetivos curriculares de la carrera con los objetivos del servicio (16).

Analizado el cruce de variables de la Tabla 3, un $42.8 \%$ de encuestados, manifiestan que sí cono- cen qué es la metodología de aprendizaje-servicio, pero indican no saber si dentro de las actividades de vinculación con la sociedad se fomenta la formación en ciudadanía activa, tampoco saben si la carrera ofrece programas para el ejercicio de la ciudadanía activa y el $64.4 \%$ mencionan que no saben sobre aprendizaje-servicio, pero a la vez consideran que sí se fomenta la formación en ciudadanía activa en las actividades de vinculación, a pesar de que la carrera no ofrece programas para el ejercicio de la ciudadanía activa.

\section{Tabla 3. Actividades de vinculación con la sociedad, formación en ciudadanía activa y conocimiento de aprendizaje-servicio (A-S)}

\begin{tabular}{|c|c|c|c|c|c|c|c|}
\hline \multirow{2}{*}{\multicolumn{4}{|c|}{ ¿Conoce que es la metodología A-S? }} & \multicolumn{3}{|c|}{$\begin{array}{l}\text { ¿La carrera ofrece programas para el } \\
\text { ejercicio de la ciudadanía activa? }\end{array}$} & \multirow{2}{*}{ Total } \\
\hline & & & & Si & No & No sabe & \\
\hline \multirow[t]{4}{*}{$\mathrm{Si}$} & \multirow{3}{*}{$\begin{array}{l}\text { ¿Se fomenta la formación en ciudadanía } \\
\text { activa? }\end{array}$} & $\mathrm{Si}$ & $\%$ & $41.7 \%$ & $40.0 \%$ & $31.9 \%$ & $33.2 \%$ \\
\hline & & No & $\%$ & $16.7 \%$ & $3.3 \%$ & $25.3 \%$ & $25.4 \%$ \\
\hline & & No sabe & $\%$ & $41.7 \%$ & $26.7 \%$ & $42.8 \%$ & $41.5 \%$ \\
\hline & Total & & & 12 & 15 & 166 & 193 \\
\hline \multirow[t]{4}{*}{ No } & \multirow{3}{*}{$\begin{array}{l}\text { ¿Se fomenta la formación en ciudadanía } \\
\text { activa? }\end{array}$} & Si & $\%$ & $65.6 \%$ & $64.4 \%$ & $46.1 \%$ & $59.5 \%$ \\
\hline & & No & $\%$ & $15.6 \%$ & $22.0 \%$ & $22.7 \%$ & $20.2 \%$ \\
\hline & & & $\%$ & $18.8 \%$ & $13.6 \%$ & $31.2 \%$ & $20.4 \%$ \\
\hline & Total & & & 154 & 191 & 141 & 486 \\
\hline \multirow{4}{*}{$\begin{array}{l}\text { No } \\
\text { Sabe }\end{array}$} & \multirow{3}{*}{$\begin{array}{l}\text { ¿Se fomenta la formación en ciudadanía } \\
\text { activa? }\end{array}$} & $\mathrm{Si}$ & $\%$ & $64.1 \%$ & $45.7 \%$ & $17.2 \%$ & $29.1 \%$ \\
\hline & & No & $\%$ & $25.6 \%$ & $20.0 \%$ & $30.7 \%$ & $28.3 \%$ \\
\hline & & No sabe & $\%$ & $10.3 \%$ & $34.3 \%$ & $52.1 \%$ & $42.6 \%$ \\
\hline & & & & 39 & 35 & 163 & 237 \\
\hline
\end{tabular}

Elaborado por autores

Fuente: Docentes, estudiantes de la Facultades de Ciencias Humanas, Ciencias de la Salud, Jurisprudencia y Ciencias Sociales

A un nivel de significancia $\alpha 0,05$, el p-valor es 0,742 , las actividades de proyección social que tienen conexión con los planes nacionales, locales y comunitarios favorecerán a la formación profesional y responsabilidad ciudadana (Figura 2).

Figura 2. Relación actividades de vinculación con los planes nacionales y locales

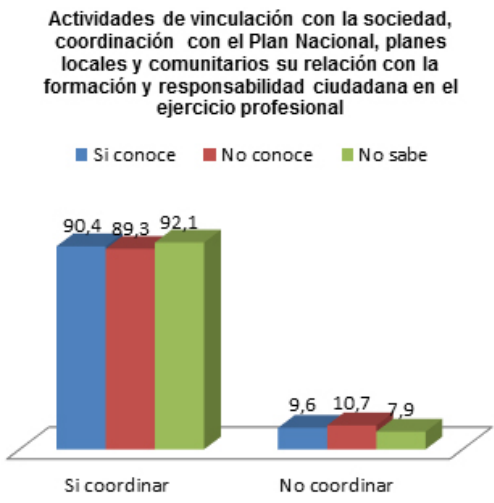

Elaborado por autores

Fuente: Docentes, y población de estudiantes de la

Facultades de Ciencias Humanas, Ciencias de la Salud,

Jurisprudencia y Ciencias Sociales.
En lo referente a si coordinar las actividades de vinculación con el Plan nacional, planes locales y comunitarios, el $90,4 \%$ sí conoce lo que implica la formación y responsabilidad ciudadana en el ejercicio profesional. Caso contrario, en relación a no coordinar las actividades de vinculación con el Plan nacional, planes locales y comunitarios el $10,7 \%$ no conoce lo que implica la formación y responsabilidad ciudadana en el ejercicio profesional.

Si el propósito de las actividades de Proyección social de la UTA es: Acompañar a las comunidades para desarrollar: conciencia, conocimiento, estructuras locales para mejorar calidad de vida mediante actividades de capacitación, diseño y ejecución de proyectos, consejería, voluntariado y servicio comunitario, es necesario tener una lectura de la realidad presente y futura en conexión directa con el Plan Nacional para el Buen Vivir (2009-2013) que en su objetivo 2 mejorar las capacidades y potencialidades de la ciudadanía en numeral 2.5 se refiere a: "Fortalecer la Educación Superior con visión científica y humanista 
articulada a los objetivos para el Buen Vivir"(17).

Creación de centros de vinculación comunitaria y su relación con la planificación de actividades de proyección social.

Al analizar los resultados de la Tabla 4 se observa que el $52 \%$ de los encuestados responden no conocer si el plan de trabajo previo al desarrollo de las actividades de vinculación es compartido con su comunidad o Institución y consideran que se podría crear un trabajo más eficiente y directo con la Universidad, al tener un Centro de Vinculación con la Sociedad en su comunidad o institución. Se identifica que uno de los problemas que afecta el desarrollo sostenible de la comunidad es la desarticulación entre el Plan Nacional de Desarrollo, planes de gobierno locales y planes comunitarios, las políticas deben ser coherentes y responder a las necesidades de las comunidades, es importante que la comunidad tenga una visión amplia que forma parte de una región territorial, que comparte de manera sistémica un ambiente ecológico, vegetación y cultivos similares, sistemas parecidos de producción, un sistema de transporte y comunicación, cierto equilibrio étnico, una identidad y algunos elementos culturales en común(17).

Tabla 4. El trabajo es más eficiente y directo al contar con un Centro de Vinculación en la comunidad, compartir el plan de trabajo y en coordinación con el Plan Nacional de Desarrollo

\begin{tabular}{|c|c|c|c|c|c|c|c|}
\hline \multicolumn{4}{|c|}{ Plan Nacional de Desarrollo } & \multicolumn{3}{|c|}{$\begin{array}{l}\text { ¿El trabajo es más eficiente } \\
\text { al tener un Centro de Vincu- } \\
\text { lación en su comunidad? }\end{array}$} & \multirow[t]{2}{*}{ Total } \\
\hline & & & & & $\mathrm{Si}$ & No & \\
\hline \multirow[t]{3}{*}{$\mathrm{Si}$} & \multirow{3}{*}{$\begin{array}{l}\text { ¿El plan de trabajo previo ejecución de } \\
\text { actividades de vinculación es compartido? }\end{array}$} & $15.3 \%$ & $\mathrm{Si}$ & $\%$ & $15.3 \%$ & $11.0 \%$ & $13.9 \%$ \\
\hline & & $52.0 \%$ & No & $\%$ & $52.0 \%$ & $59.5 \%$ & $54.4 \%$ \\
\hline & & $32.7 \%$ & A veces & $\%$ & $32.7 \%$ & $29.5 \%$ & $31.7 \%$ \\
\hline & Total & & & & 563 & 264 & 827 \\
\hline \multirow[t]{4}{*}{ No } & \multirow{3}{*}{$\begin{array}{l}\text { ¿El plan de trabajo previo ejecución de } \\
\text { actividades de vinculación es compartido? }\end{array}$} & $9.7 \%$ & $\mathrm{Si}$ & $\%$ & $9.7 \%$ & $7.4 \%$ & $9.0 \%$ \\
\hline & & $58.1 \%$ & No & $\%$ & $58.1 \%$ & $51.9 \%$ & $56.2 \%$ \\
\hline & & & A veces & $\%$ & $32.3 \%$ & $40.7 \%$ & $34.8 \%$ \\
\hline & Total & & & & 62 & 27 & 89 \\
\hline
\end{tabular}

Elaborado por autores

Fuente: Docentes, estudiantes de la Facultades de Ciencias Humanas, Ciencias Sociales, Jurisprudencia y Ciencias Sociales

A un nivel de significancia a 0,05, el p-valor es 0,131 , la creación de los centros de vinculación con la sociedad en la comunidad ayudará a mejorar el plan de trabajo, previo la ejecución de actividades de proyección social (Figura 3) afirmándose con (Antiguo verso chino). Ve en busca de Tu Gente Ámalos, aprende de ellos, planea con ellos, sírveles, empieza con lo que tienen, básate en que saben. Pero de los mejores líderes cuando su tarea se realiza, su trabajo se termina toda la gente comenta: lo hemos hecho nosotros mismos.

Figura 3. Planes de trabajo y su relación con centros de vinculación

Creación de un centro de Vinculación con la Sociedad en las comunidades y la elaboración conjunta del plan de trabajo previo al desarrollo de las actividades

- Si compartido No compartido a veces

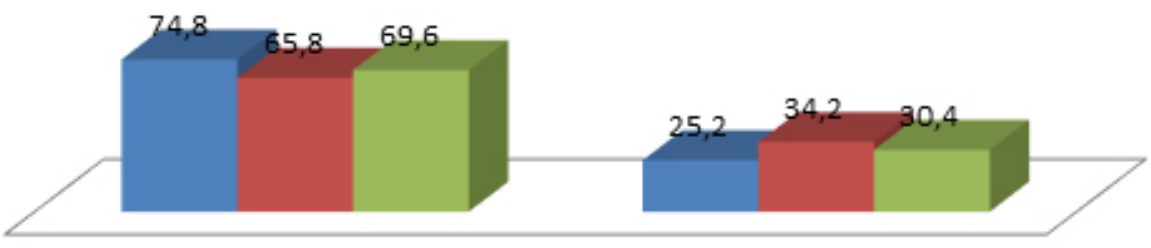

Si considera

No considera

Elaborado por autores

Fuente: Docentes, estudiantes de la Facultades de Ciencias Humanas, Ciencias Sociales, Jurisprudencia y Ciencias Sociales 


\section{Conclusiones}

Se evidencia la necesidad de establecer condiciones que hagan de las actividades de vinculación con la sociedad un proceso formal, estructurado y reflexivo, siendo para ello necesario, diarios de campo, informes periódicos, diagnósticos, sistematización de experiencias y un sistema de evaluación específico.

Deficiente integración y coordinación de los docentes de la universidad y las autoridades locales, no existe un plan de trabajo previo para el cumplimiento de las actividades de proyección social. Hay limitaciones de herramientas en su formación pedagógica para enfrentar el reto de las actividades de vinculación con la sociedad. La mayoría de los estudiantes no recibieron información ni preparación pertinente sobre temas que fortalezcan la ciudadanía activa y habilidades sociales dentro de su formación lo que incide en su desempeño profesional.

Es manifiesta la importancia de recibir, dentro de su formación, aspectos que fortalezcan la competencia de Responsabilidad Social (educación para la vida en sociedad, prosocialidad, resiliencia) factor importante dentro del perfil profesional.

En cuanto a la metodología de aprendizaje-servicio y el fortalecimiento de la ciudadanía activa, manifiestan no estar insertos en estos contenidos programáticos. Las carreras fomentan en parte la participación de alumnos y profesores en planes de desarrollo y a veces el plan de actividades es compartido con la comunidad, mencionan que en las carreras se podrían articular las prácticas pre profesionales, vinculación con la comunidad y el proyecto de tesis de graduación, como parte del programa de aprendizaje servicio.

En la elaboración de proyectos de vinculación no existe articulación con los planes de desarrollo local y nacional, se elaboran solo desde la Universidad y no satisfacen las necesidades reales de las comunidades o instituciones. Los estudiantes mencionan que al realizar estas actividades consiguieron conocer el lugar de actuación, su mayor dificultad en su limitada experiencia, siendo necesario fortalecer conocimientos significativos de utilidad para la vida, mejorar la formación específica en cuanto a metodologías participativas, capacitación de adultos, educación popular y otras relacionadas con el efectivo desempeño del alumno en la comunidad.

Se necesita un fin trascendente, una bondad sostenible que se espera al final de la intervención como resultado de su trabajo, priorizar la elabora- ción de un proyecto sostenible, sistematización de experiencias y publicación de resultados.

El propósito de la Universidad es promover la adquisición y generación de conocimientos, al contar con un centro universitario en la comunidad, se conocería mejor la realidad de la región territorial que comparten las mismas características de clima, tierra y formas de producción y personas de diferentes etnias guiadas por los programas de la universidad pueden trabajar juntos para aumentar sus conocimientos. Es importante comprender la organización territorial a la cual pertenecen como una unidad sistémica. En general, lo que hace falta es una planificación concertada y una coordinación interinstitucional, a nivel regional, esto ayudará a evitar la duplicación de esfuerzos a crear un impacto sinérgico y ampliar la oferta de programas a todas las comunidades que tienen la voluntad de responsabilizarse por su propio desarrollo. Lo cierto es, que aún falta consolidar un "norte" que vincule educación, desarrollo nacional y necesidad popular como una totalidad sistémica. Una propuesta sistémica desarticulada, sumada a una tradición de débil anclaje territorial ha producido un divorcio universidad-sociedad, en el que el aislamiento se ha perversamente naturalizado.

Se observa que el sistema educativo promueve activamente la cosmovisión del agonismo, mediante los textos impresos, las lecciones impartidas por los educadores, el sistema de evaluación comparativa, el fomento de deportes competitivos, e incluso la cultura de constantes pugnas al interior de la comunidad educativa, que incluye a estudiantes, padres de familia, educadores y administradores. Si se agrega a estos factores la violencia transmitida por los medios masivos y los modelos que nos presentan los sistemas partidistas, económico y judicial, es evidente que nos encontramos sumergidos en una cultura del agonismo desde la cuna hasta el sepulcro. Estas no son objeciones a la cultura de paz, sino señales de cuánto hace falta el enfoque pedagógico de aprendizaje servicio como instrumento para promoverla por todos los medios.

\section{Referencias Bibliográficas}

1. Naranjo Claudio. Cambiar la educación para cambiar el mundo. Primera ed. llave L, editor. Chile: La llave; 2011.

2. C. HWyRdF. Developing the Whole Student. Primera ed. Unido R, editor. Londres: Reino Unido; 2011.

3. J R. La civilización empàtica. Primera ed. Pai- 
dós, editor. Barcelona: Paidós; 2010.

4. Rama C. La tercera Reforma de la educación superior en América Latina. Educación y Pedagogía. 2006 Noviembre; XVIII(46).

5. G TR. Comprtitividad en la Educación Superior Tecnológica. Avance Tecnológico Cultural, Conocimiento y divulgación. 2011 Junio; 5(3).

6. G. M. Currículo por Competencias. Primera ed. Salvador A, editor. Colombia: Salvador Allende; 2011.

7. L.E. O. Calidad Académica y Relevancia Social de la Educación Superior en America Latina. Revista Iberoamericana de Educación Superior. 2010 Abril; 1(1).

8. S. C. El $8^{\mathrm{a}}$ hábito de la efectividad a la grandeza. Primera ed. Paidos I, editor. Colombia: Paidos Iberica; 2005.

9. J. AE\&H. Participación Comunitaria. Primera ed. Abya , editor. Santa Cruz-Bolivia: Abya; 2010.

10. Rico S. Educación Superior e Innovación. Diseño Curricular por competencias para la formación en el siglo XXI. Primera ed. Académica E, editor. Germany: Académica Española; 2012.

11. R. H. Educación Superior Cambios de fondo. Vistazo. 2009 Febrero; 03(20).

12. L.H. SV. Participacion ciudadana y movimientos sociales. Universidad de Lauvain. 2010 Agosto; $1(1)$.

13. Zurita S. Aprendizaje Servicio. CONVERSACION SOBRE LOS TEMAS DE APRENDIZAJE. 2007 Marzo; 1(1).

14. SENPLADES. El Plan Nacional paea el Buen Vivir. Segundo ed. conejo E, editor. Quito: El conejo; 2010.

15. F. C. Sociedad Educatora. Iberoamericana. 2001 Agosto; 26(26).

16. M. T. Aprendizaje y Servicio Solidario en America Latina. Programa Nacional Educación Solidaria. 2006 Agosto; 1(1).

17. SENPLADES. Plan Nacional del Buen Vivir. Segunda ed. conejo e, editor. Quito: El conejo; 2010. 